\title{
MiR-361-5p suppresses chemoresistance of gastric cancer cells by targeting FOXM1 via the PI3K/Akt/mTOR pathway
}

\author{
Lei Tian ${ }^{1, *}$, Zhifeng Zhao ${ }^{2, *}$, Ling $X^{3}{ }^{3}$ and JinPeng Zhu ${ }^{1}$ \\ ${ }^{1}$ Department Gastroenterol, Jinzhou Medical University, Affilliated Hospital 1, Jinzhou 121000, Liaoning Province, Peoples \\ Republic of China \\ ${ }^{2}$ Department Gastroenterol, Zhongguo Medical University, Affilliated Hospital 4, Shengyang 110000, Liaoning Province, \\ Peoples Republic of China \\ ${ }^{3}$ Department Anatomy, Jinzhou Medical University, Jinzhou 121000, Liaoning Province, Peoples Republic of China \\ *These authors have contributed equally to this work and should be considered co-first authors \\ Correspondence to: Lei Tian, email: tianleiln@163.com \\ Keywords: MiR-361-5p; autophagy; chemoresistance; FOXM1; PI3K/AKt/mTOR \\ Received: October 17, $2017 \quad$ Accepted: December 05, $2017 \quad$ Published: December 20, 2017 \\ Copyright: Tian et al. This is an open-access article distributed under the terms of the Creative Commons Attribution License 3.0 \\ (CC BY 3.0), which permits unrestricted use, distribution, and reproduction in any medium, provided the original author and source \\ are credited.
}

\section{ABSTRACT}

Gastric cancer is a prevalent cancer and chemotherapy is a main treatment for patients. Docetaxel is commonly used as a chemotherapeutic drug for gastric cancer patients. With the increasing emergence of docetaxel resistance, exploring the mechanism of chemoresistance may improve prognosis of patients. In this study, we found that overexpressed miR-361-5p suppressed chemoresistance to docetaxel of gastric cancer cells (SGC-7901, MKN-28) by decreasing IC $_{50}$ values of docetaxel while increasing cell apoptosis rate, especially in docetaxel resistant SGC-7901 cells. Further researches revealed that overexpressed miR-361-5p inhibited chemoresistance through inhibiting autophagy with a characteristic of declined number of LC3+ puncta, decreased expression of Beclin-1 and the ratio of LC3 II/I and increased expression of p62. Bioinformatics study and Luciferase reporter assay indicated that FOXM1 was a target of miR-361-5p and FOXM1 was negatively regulated by miR-361-5p in gastric cancer. Simultaneously, overexpression of FOXM1 counteracted the inhibitory effects of miR-361-5p on chemoresistance of gastric cancer cells through activating autophagy, further certifying the targeting relationship between the two. Moreover, overexpressed miR-361-5p activated the PI3K/Akt/mTOR pathway. The adding of PI3K inhibitor LY294002 played an opposite role to miR-361-5p mimic by inducing autophagy and chemoresistance to docetaxel of gastric cancer cells compared with docetaxel + miR-361-5p mimic group, indicating that miR-361-5p suppressed autophagy-induced chemoresistance via the PI3K/Akt/mTOR pathway in gastric cancer cells. In conclusion, we found that miR-361-5p suppressed autophagy-induced chemoresistance of gastric cancer cells through targeting FOXM1 via the PI3K/Akt/ mTOR pathway, providing a foundation for the mechanism research and treatment of gastric cancer.

\section{INTRODUCTION}

Gastric cancer (GC) is one of the most prevalent cancers around the world, accounting for almost $10 \%$ of all cancer death [1]. Because the clinical symptom is not obvious at early stage, patients with $\mathrm{GC}$ are often detected at late stage, thus causing an extremely low 5-year survival rate [2]. Surgery is considered as the main treatment for patients with GC. However, for patients who miss the best time for surgery, palliative 
chemotherapy is the main choice of treatment. During the past decades, several new-generation cytotoxic agents including docetaxel have been used for the treatment of GC [3]. Docetaxel has been reported to promote the assembly and stabilization of microtubules to inhibit the depolymerization in the treatment of GC [4, 5]. However, obvious resistance to docetaxel has been reported in some types of tumors including prostate cancer, breast cancer, GC and nasopharyngeal carcinoma, seriously limiting the treatment effect of docetaxel [4, 6-9]. Therefore, inhibiting chemoresistance to docetaxel in GC may be a key role for improving therapeutic effects for GC patents.

Autophagy is a cellular process which delivers intracellular aggregated or misfolded proteins to the lysosomal compartment where they are degraded and recycled [10]. Previous studies reported that activation of autophagy enhanced chemoresistance in various cancers. Fukuda's study indicated that inhibition of autophagy suppressed proliferation and overcame Cisplatin resistance of endometrial cancer cells [11]. A study by Zhang revealed that inhibition of TRPC5-induced autophagy suppressed drug resistance to Adriamycin in breast carcinoma [12]. However, detailed contributions of autophagy to chemoresistance of GC cells are still limited and require for further investigations.

MicroRNAs (miRNAs), a group of small non-coding RNAs with 20-22 nucleotides, are reported to regulate the expression of specific genes [13]. Accumulated evidence indicated that miRNAs were involved in a great number of cellular processes including proliferation, apoptosis, migration and invasion during tumor progression $[14,15]$. Previous studies showed that some miRNAs such as miR608 and miR-204 suppressed chemoresistance of tumor cells $[16,17]$. MiR-361-5p, one of the miRNAs, was found to function as a tumor suppressor in various tumors. Previous study showed that overexpressed miR-361-5p inhibited tumor growth of hepatocellular carcinoma in nude mice significantly [18]. However, whether miR$361-5 p$ can regulate chemoresistance of tumor cells is still unclear.

MiRNAs are reported to regulate the expression of their target genes to accomplish their effects. In our presents study, we found that FOXM1, a newly unified family member of Forkhead transcription factor, was one of the target genes of miR-361-5p [19]. FOXM1 was reported to confer resistance to herceptin and paclitaxel by altering microtubule dynamics to protect tumor cells from paclitaxel-induced apoptosis in breast cancer [20]. As both docetaxel and FOXM1 affected the microtubules dynamics, we suggested that FOXM1 might involve in chemoresistance to docetaxel, however, little research has been carried out.

In our present study, we explored the mechanism of miR-361-5p/FOXM1 axis in regulating the chemoresistance of GC cells. We found that miR-361-5p suppressed autophagy-induced chemoresistance of GC cells through targeting FOXM1 via the PI3K/Akt/mTOR pathway, trying to find a new direction for the treatment of GC.

\section{RESULTS}

\section{MiR-361-5p suppresses chemoresistance of gastric cancer cells to docetaxel}

We initiated our study by investigating whether there was an association between miR-361-5p and chemoresistance to docetaxel of GC cells. SGC-7901 cells which were resistant to docetaxel and MKN-28 cells which were sensitive to docetaxel were treated with different concentrations of docetaxel and then cell viability was detected by MTT assay. We observed that docetaxel worked as an anti-tumor regent by suppressing cell viability significantly in a dose-dependent manner and the cell viability of SGC-7901 was higher than docetaxel sensitive cell line MKN-28 under the same concentration of docetaxel. Moreover, the half maximal inhibitory concentration $\left(\mathrm{IC}_{50}\right)$ values of docetaxel were approximately $0.025 \mathrm{mg} / \mathrm{L}$ and $0.015 \mathrm{mg} / \mathrm{L}$ in $\mathrm{miR}-361$ $5 p$ mock transfected SGC-7901 cells and miR-361-5p mimic transfected SGC-7901 cells, respectively. However, the $\mathrm{IC}_{50}$ in miR-361-5p mock transfected and miR-361$5 \mathrm{p}$ mimic transfected MKN-28 cells were approximately $0.015 \mathrm{mg} / \mathrm{L}$ and $0.013 \mathrm{mg} / \mathrm{L}$ respectively. These results suggested that overexpression of miR-361-5p increased the sensibility to docetaxel of gastric cancer cells and the promoting role was more obviously in docetaxel resistant cells (Figure 1A-1B). Apart from that, results from cell apoptosis assay indicated that overexpression of miR-361-5p increased cell apoptosis rate compared with docetaxel + mock group in both docetaxel treated SGC7901 and MKN-28 cells (Figure 1C-1D, $P^{*}<0.05, P^{\#}<$ 0.05). Docetaxel resistant cells SGC-7901 were chosen for following experiments. In conclusion, our results suggested that overexpressed miR-361-5p inhibited chemoresistance of GC cells to docetaxel.

\section{Overexpressed miR-361-5p suppresses chemoresistance of gastric cancer cells by inhibiting autophagy}

Accumulating evidence supported that chemoresistance of cancer cells was correlated with autophagy [21]. Therefore, we investigated whether miR-361-5p suppressed chemoresistance of GC through autophagy in our present study. The localization of LC3 to autophagosome formation was assessed through GFPLC3 expression. Our data showed that the number of $\mathrm{LC}^{+}$ puncta significantly increased after docetaxel treatment compared with control group. However, overexpressed miR-361-5p reduced the number of $\mathrm{LC}^{+}$puncta induced by docetaxel treatment compared with docetaxel + mock 
group markedly (Figure 2A-2B, $P^{* *}<0.01, P^{\#}<0.05$ ). Then the expression of autophagy-related proteins was measured by western blot. Docetaxel treatment increased the expression of Beclin-1 and the ratio of LC3 II/I while decreased the expression of p62 compared with control group. Overexpressed miR-361-5p decreased the expression of Beclin-1 and the ratio of LC3 II/I while increased the expression of p62 compared withdocetaxel + mock group significantly, suggesting that miR-361-5p suppressed chemoresistance of GC cells through inhibiting autophagy (Figure 2C-2D, $P^{* *}<0.01, P^{\#}<0.05$ ). In order to verify our conjecture, autophagy inducing reagent rapamycin (Rapa) was used in our study. Because Rapa was dissolved in DMSO, thus DMSO group was used as control and we found that DMSO didn't have significant effect on cell viability and apoptosis rate. We observed that the $\mathrm{IC}_{50}$ values were approximately $0.015 \mathrm{mg} / \mathrm{L}$ and 0.025 $\mathrm{mg} / \mathrm{L}$ in miR-361-5p mimic group and mimic + Rapa group respectively, indicating that activation of autophagy by Rapa decreased the sensibility to docetaxel of overexpressed miR-361-5p GC cells (Figure 2E). Besides that, activation of autophagy abolished the promoting effect of miR-361-5p on cell apoptosis compared with docetaxel + mimic group remarkably (Figure $2 \mathrm{~F}, P^{*}<$ $\left.0.05, P^{\#}<0.05\right)$, suggesting that activation of autophagy increased chemoresistance of GC cells to docetaxel. Taken together, these results supported that overexpressed miR361-5p suppressed chemoresistance of GC cells through inhibiting autophagy.

\section{FOXM1 is a target of miR-361-5p in gastric cancer cells}

In order to explore the molecular mechanisms of miR-361-5p in chemoresistance of GC cells, putative miR-361-5p targets were predicted through bioinformatics analysis. The predicted results showed that FOXM1 was one of the potential targets of miR-361-5p (Figure 3A). SGC-7901 cells were transfected with pcDNA3.1-FOXM1 for overexpression of FOXM1 (Figure 3B). Results from western blot showed that the expression of FOXM1 was increased by docetaxel treatment compared with control group and was decreased by the adding of miR-361-5p mimic compared with pcDNA3.1-FOXM1 group under the application of docetaxel, indicating that the expression of FOXM1 was negatively regulated by miR-361-5p (Figure $3 \mathrm{C}-3 \mathrm{D}, P^{*}<0.05, P^{\#}<0.05, P^{\$}<0.05$ ). Moreover, the luciferase reporter assay showed that co-transfection with miR-361-5p mimic and FOXM1 WT in SGC-7901 cells led to a significant decrease in luciferase activity.
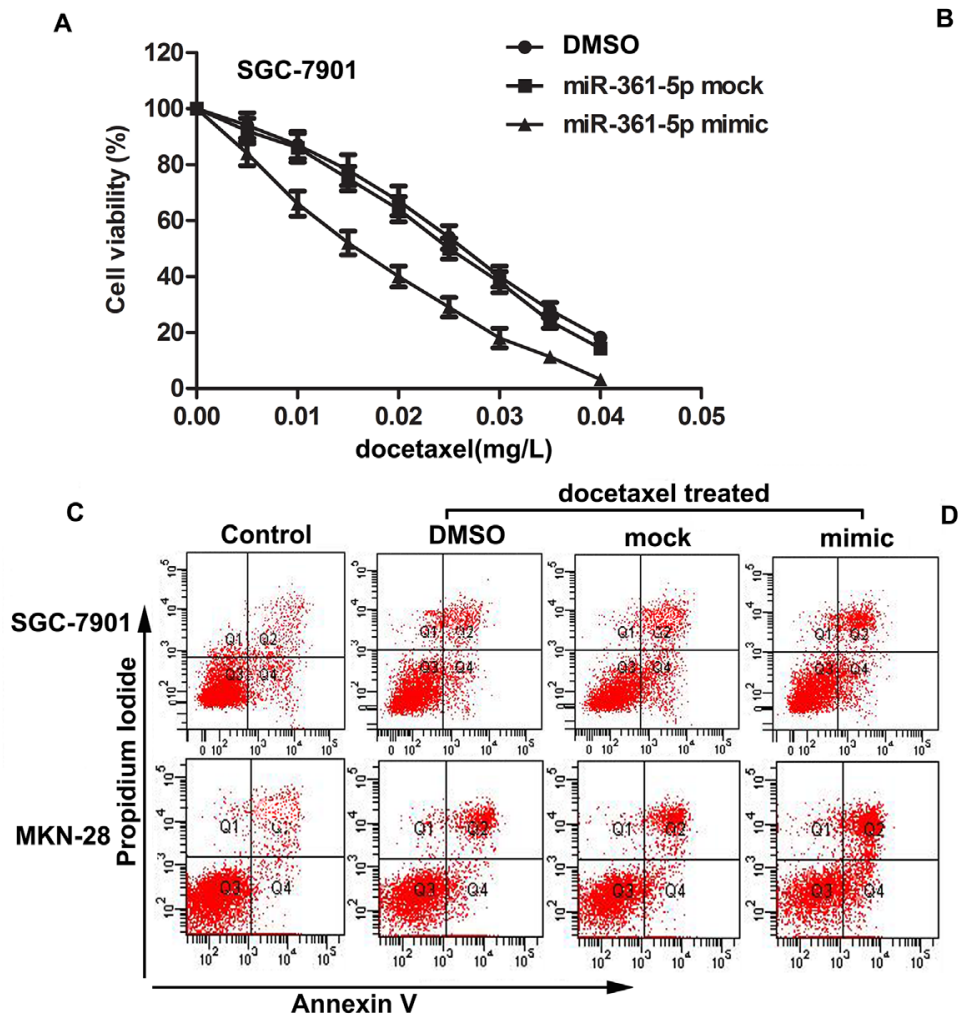

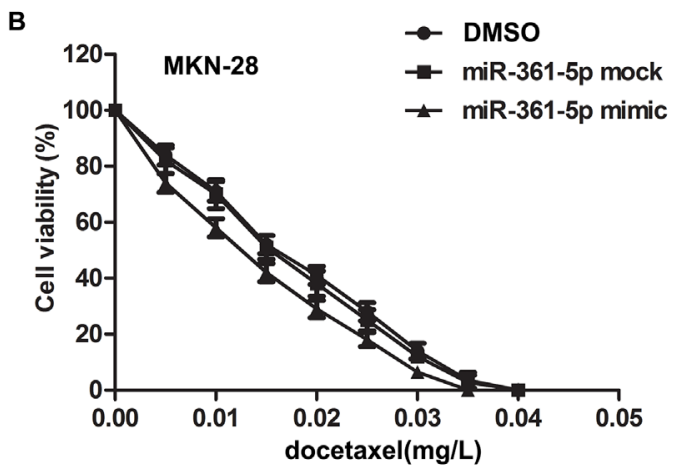

D

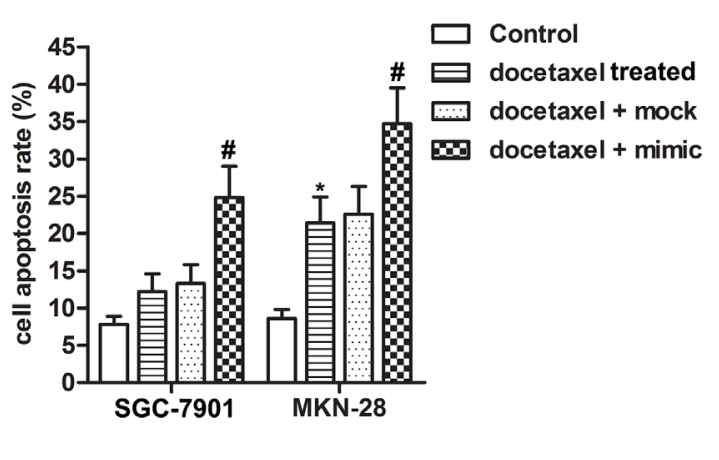

Figure 1: MiR-361-5p suppresses chemoresistance of gastric cancer cells to docetaxel. GC cells were transfected with miR-361-5p mimic or miR-361-5p mock respectively. (A-B) Cell viability of transfected GC cells was tested by MTT assay. (C-D) Cell apoptosis was detected by flow cytometric analysis. The bars showed means $\pm \mathrm{SD}$ of three independent experiments. $P^{*}<0.05$ compared with control group, $P^{\#<0.05}$ compared with docetaxel + mock group. 
However, co-transfection with FOXM1 MUT and miR361-5p mimic didn't show significant difference compared with cells transfected with FOXM1 MUT (Figure $\left.3 \mathrm{E}, P^{* *}<0.01\right)$, confirming the targeting relationship between FOXM1 and miR-361-5p. In summary, our data elucidated that FOXM1 was a target of miR-361-5p and the expression of FOXM1 was negatively regulated by miR-361-5p in GC cells.

\section{Overexpression of FOXM1 decreases the inhibitory effects of miR-361-5p on autophagy- induced chemoresistance in gastric cancer cells}

Having learned the relationship between FOXM1 and miR-361-5p, we then set to explore the mechanism of miR-361-5p in chemoresistance of GC cells. Co- transfection with miR-361-5p mimic and pcDNA3.1FOXM1 increased the number of $\mathrm{LC}^{+}$puncta, the expression of Beclin-1 and the ratio of LC3 II/I while decreased the expression of p62 compared with docetaxel + mimic group, indicating that overexpressed FOXM1 weaken the inhibitory effect of miR-361-5p on autophagy (Figure 4A-4D, $P^{*}<0.05, P^{* *}<0.01, P^{\#}<0.05, P^{\$}<$ $0.05)$. Besides that, our data showed that $\mathrm{IC}_{50}$ values were approximately $0.015 \mathrm{mg} / \mathrm{L}$ and $0.025 \mathrm{mg} / \mathrm{L}$ in $\mathrm{miR}-361-5 \mathrm{p}$ mimic group and mimic + FOXM1 group respectively and overexpressed FOXM1 inhibited cell apoptosis compared with docetaxel + mimic group remarkably, suggesting that overexpressed FOXM1 counteracted the inhibitory effect of miR-361-5p on chemoresistance of GC cells (Figure 4E-4F, $\left.P^{*}<0.05, P^{\#}<0.05, P^{\$}<0.05\right)$. Taken together, these data clearly indicated that overexpression of
A

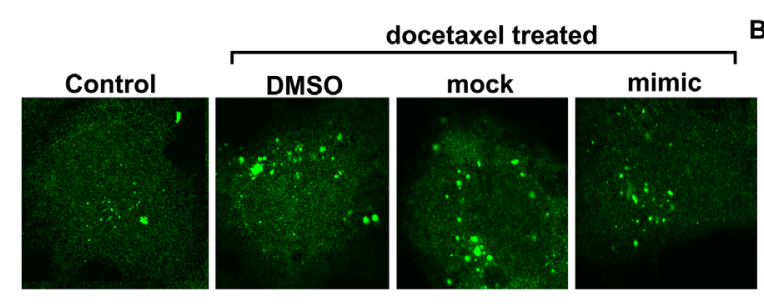

C

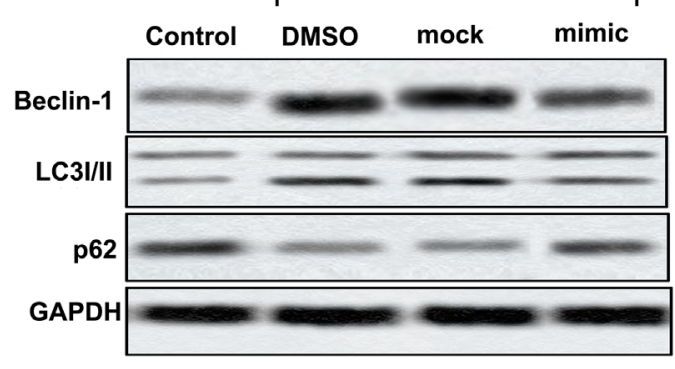

E

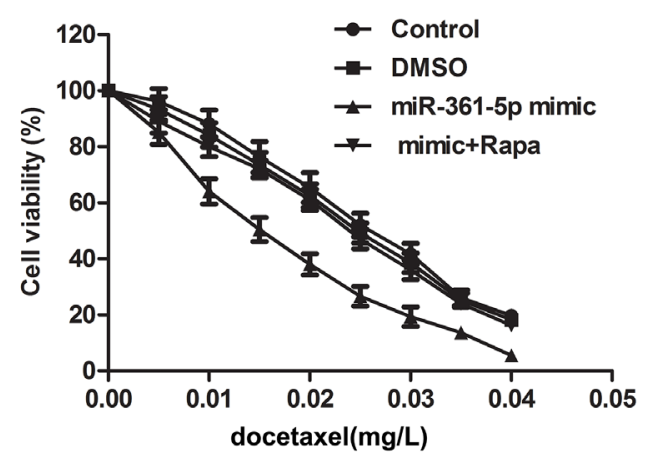

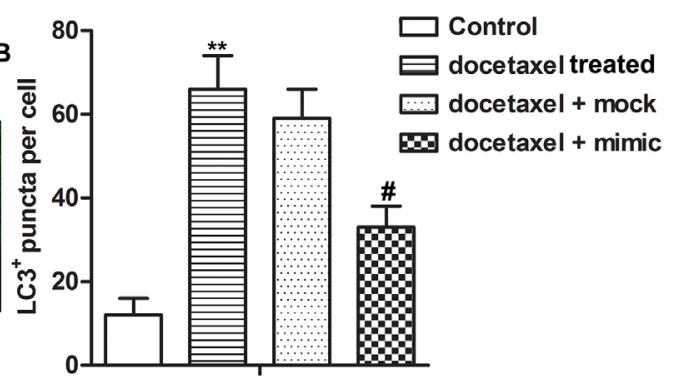

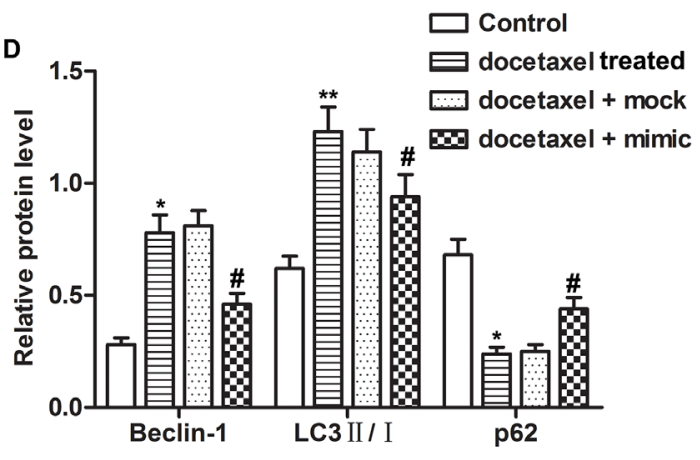

$\mathbf{F}$

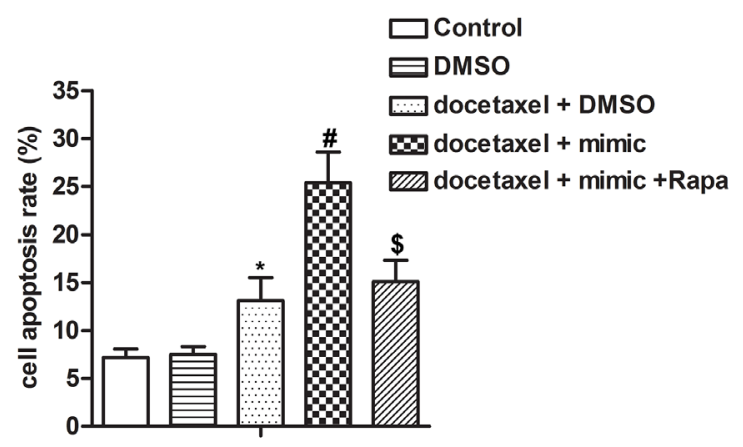

Figure 2: Overexpressed miR-361-5p suppresses chemoresistance of gastric cancer cells by inhibiting autophagy. SGC7901 transfected with miR-361-5p mimic or miR-361-5p mock respectively and received docetaxel treatment or DMSO treatment alone. (A-B) LC3 ${ }^{+}$puncta per cell was detected through fluorescence microscopy. (C-D) Relative expression of Beclin-1, LC3 I/II and p62 was detected by western blot. GAPDH was used as an endogenous reference. (E) Cell viability was tested by MTT assay. (F) Cell apoptosis was detected by flow cytometric analysis. The bars showed means $\pm \mathrm{SD}$ of three independent experiments. $P^{*}<0.05, P^{* *}<0.01$ compared with control group, $P^{\#<0.05}$ compared with docetaxel + mock group, $P^{\S}<0.05$ compared with docetaxel + mimic group. 
FOXM1 counteracted the inhibitory effects of miR-361$5 \mathrm{p}$ on autophagy-induced chemoresistance in GC cells.

MiR-361-5p suppresses autophagy-induced chemoresistance via the PI3K/Akt/mTOR pathway

Previous study reported that PI3K/Akt/mTOR pathway played an important role in regulating autophagy [22]. Therefore, we further explored whether miR-361-5p suppressed chemoresistance of GC cells through PI3K/ Akt/mTOR pathway. The data revealed that docetaxel treatment decreased the expression of p-AKT and mTOR compared with control group. However, overexpression of miR-361-5p increased the low expression of p-AKT and $\mathrm{mTOR}$ caused by docetaxel while overexpressed FOXM1 decreased the expression of p-AKT and mTOR significantly compared with docetaxel + miR-361-5p mimic group, suggesting that overexpression of miR-361$5 \mathrm{p}$ activated while overexpressed FOXM1 suppressed

\section{A Position 750-757 of FOXM1 3' UTR $\quad 5^{\prime}$...GCUAUUAGAUGUUUCUCUGAUAA... hsa-miR-361-5p 3' CAUGGGGACCUCUAAGACUAUU}

B

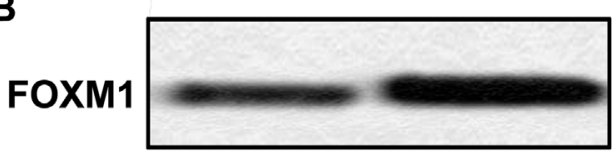

GAPDH

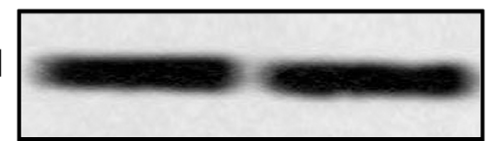<smiles>COCCOCCO</smiles>

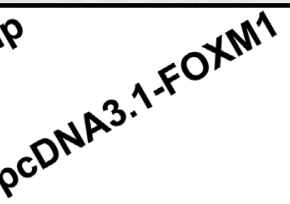

D

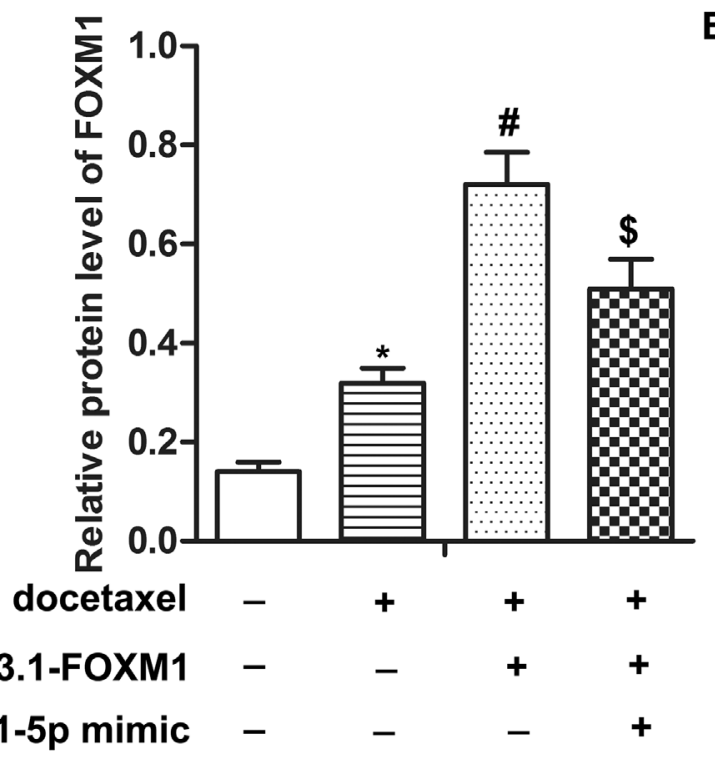

C

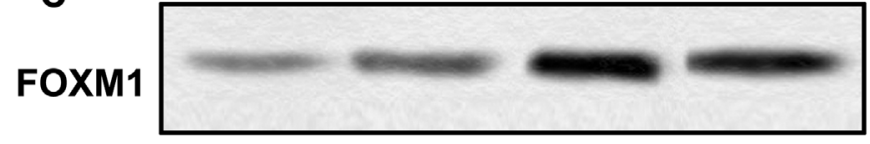

GAPDH

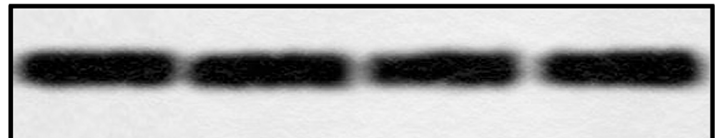

docetaxel PcDNA3.1-FOXM1 miR-361-5p mimic

E
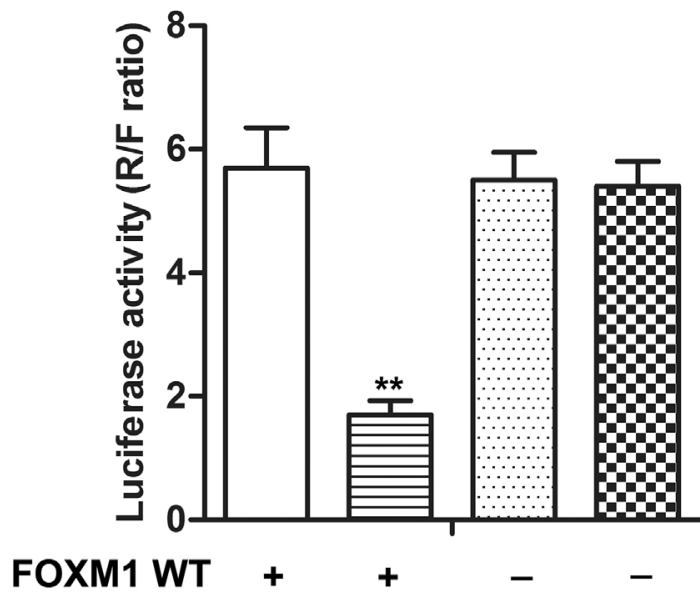

FOXM1 MUT - $\quad+\quad+$

miR-361-5p mimic $\quad-\quad+\quad-\quad+$

Figure 3: FOXM1 is a target of miR-361-5p in gastric cancer cells. (A) Bioinformatics analysis of miR-361-5p and FOXM1. (B) Relative protein level of FOXM1 in pcDNA3.1-FOXM1 group and control group was detected by western blot. GAPDH was used as an endogenous reference. (C-D) Relative protein level of FOXM1 of transfected SGC-7901 with docetaxel treatment in different groups was detected by western blot. GAPDH was used as an endogenous reference. (E) Results of Luciferase reporter assay. The bars showed means \pm SD of three independent experiments. $P^{*}<0.05, P^{* *}<0.01$ compared with control group, $P^{\#}<0.05$ compared with docetaxel treated group, $P^{\S}<0.05$ compared with docetaxel + FOXM1 group. 
$\mathrm{PI} 3 \mathrm{~K} / \mathrm{Akt} / \mathrm{mTOR}$ pathway in docetaxel treated GC cells (Figure 5A-5B, $P^{*}<0.05, P^{\#}<0.05, P^{\$}<0.05$ ). Moreover, treatment with PI3K inhibitor LY294002 increased the ratio of LC3 II/I compared with docetaxel + miR-361-5p mimic group (Figure 5C-5D, $P^{*}<0.05, P^{\#}<0.05, P^{\$}<$ $0.05)$. The $\mathrm{IC}_{50}$ values were about $0.015 \mathrm{mg} / \mathrm{L}$ and 0.025 $\mathrm{mg} / \mathrm{L}$ in miR-361-5p mimic group and mimic + LY29400 group respectively, indicating that the inactivation of $\mathrm{PI} 3 \mathrm{~K} / \mathrm{Akt} / \mathrm{mTOR}$ pathway decreased sensibility to docetaxel in GC cells transfected with miR-361-5p mimic (Figure 5E). LY294002 treatment also decreased cell apoptosis rate compared with docetaxel + miR-361$5 \mathrm{p}$ mimic group (Figure 5F, $P^{*}<0.05, P^{\#}<0.05, P^{\$}<$ $0.05)$. In conclusion, our results indicated that miR-361$5 \mathrm{p}$ suppressed autophagy-induced chemoresistance via the $\mathrm{PI} 3 \mathrm{~K} / \mathrm{Akt} / \mathrm{mTOR}$ pathway.

\section{DISCUSSION}

In gastric cancer treatment, surgery combined with chemotherapy is one of the most common methods to improve prognosis of patients. Docetaxel, one of the chemotherapy agents, is commonly used as a single agent or in combination with other agents such as latinum and fluoropyrimidine (DCF regimen) for the treatment of cancer patients $[23,24]$. Docetaxel is an active agent in advanced GC and it has also been shown to lack crossresistance with other drugs in GC [5]. However, the resistance to docetaxel did occur and more investigations on the mechanism of chemoresistance to docetaxel of tumor cells are urgently required. Our study presented the role of miR-361-5p/FOXM1 axis in the chemoresistance to

A

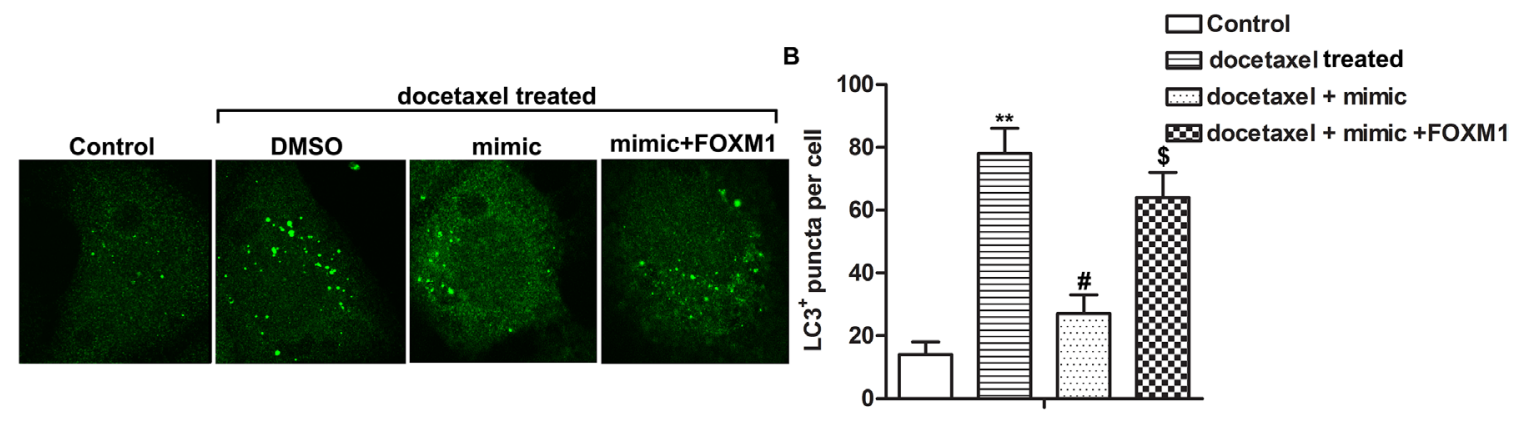

C

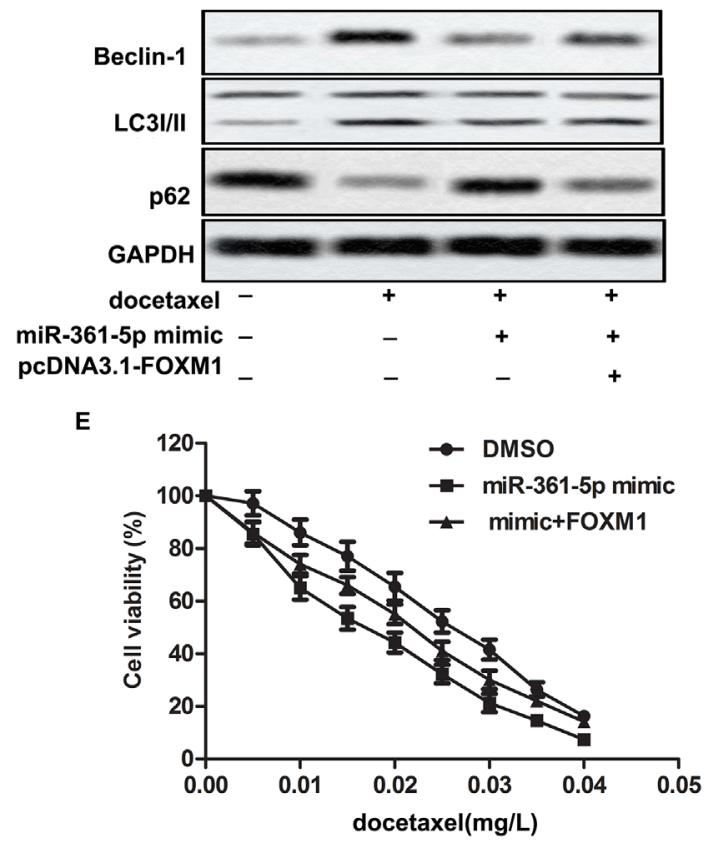

D

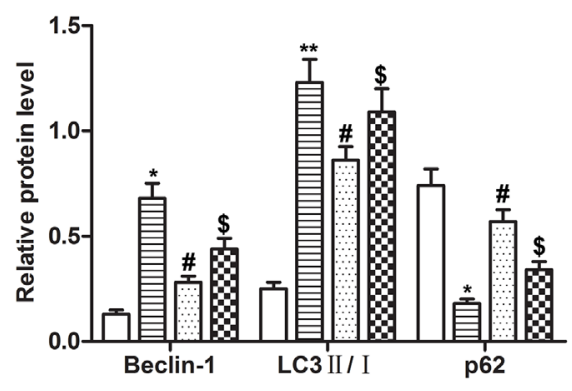

$\mathbf{F}$

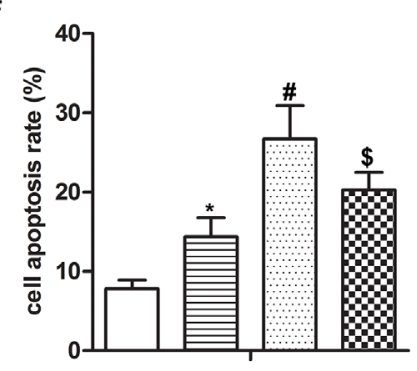

Figure 4: Overexpression of FOXM1 decreases the inhibitory effects of miR-361-5p on autophagy-induced chemoresistance in gastric cancer cells. SGC-7901 cells transfected with miR-361-5p mimic or mimic + pcDNA3.1-FOXM1 received docetaxel treatment. (A-B) $\mathrm{LC}^{+}$puncta per cell was detected through fluorescence microscopy. (C-D) Relative expression of Beclin-1, LC3 I/II and p62 was detected by western blot. GAPDH was used as an endogenous reference. (E) Cell viability was tested by MTT assay. (F) Cell apoptosis was detected by flow cytometric analysis. The bars showed means \pm SD of three independent experiments. $P^{*}<0.05, P^{* *}<0.01$ compared with control group, $P^{\#}<0.05$ compared with docetaxel treated group, $P^{\$}<0.05$ compared with docetaxel + mimic group. 
docetaxel of GC cells and tried to find a better therapeutic strategy for overcoming the resistance to docetaxel in GC.

MiRNAs have gained increasing attentions as they appeared to be involved in tumor progression. Increasing evidence supported that miRNAs acted as tumor suppressors or oncogenes and involved in post-translational regulation of gene expression [25]. Previous studies reported that a great number of miRNAs were related to chemoresistance in various cancers. For example, Rajabpour's study revealed that miR608 suppressed gemcitabine chemoresistance through regulating the resistance genes in pancreatic cancer cells [16]. Besides that, microRNA-204 was reported to increase chemosensitivity of prostate cancer cells [17]. Recently, accumulating evidence supported that miR-361-
$5 p$ acted as a tumor suppressor in many kinds of tumors. Zhuang's research indicated that down-regulation of miR361-5p associated with aggressive clinicopathological features and unfavorable prognosis in non-small cell lung cancer [26]. It was also reported that miR-361-5p inhibited epithelial-to-mesenchymal transition through targeting Twist 1 in glioma cells [27]. However, whether miR-361-5p could regulate chemoresistance of tumor cells still remained unknown. In our present study, SGC-7901 cells which were resistant to docetaxel and MKN-28 cells which were sensitive to docetaxel were chosen for our experiments. Results indicated that overexpressed miR361-5p in SGC-7901 and MKN-28 cells decreased the $\mathrm{IC}_{50}$ value of docetaxel and increased cell apoptosis rate, suggesting that miR-361-5p inhibited chemoresistance
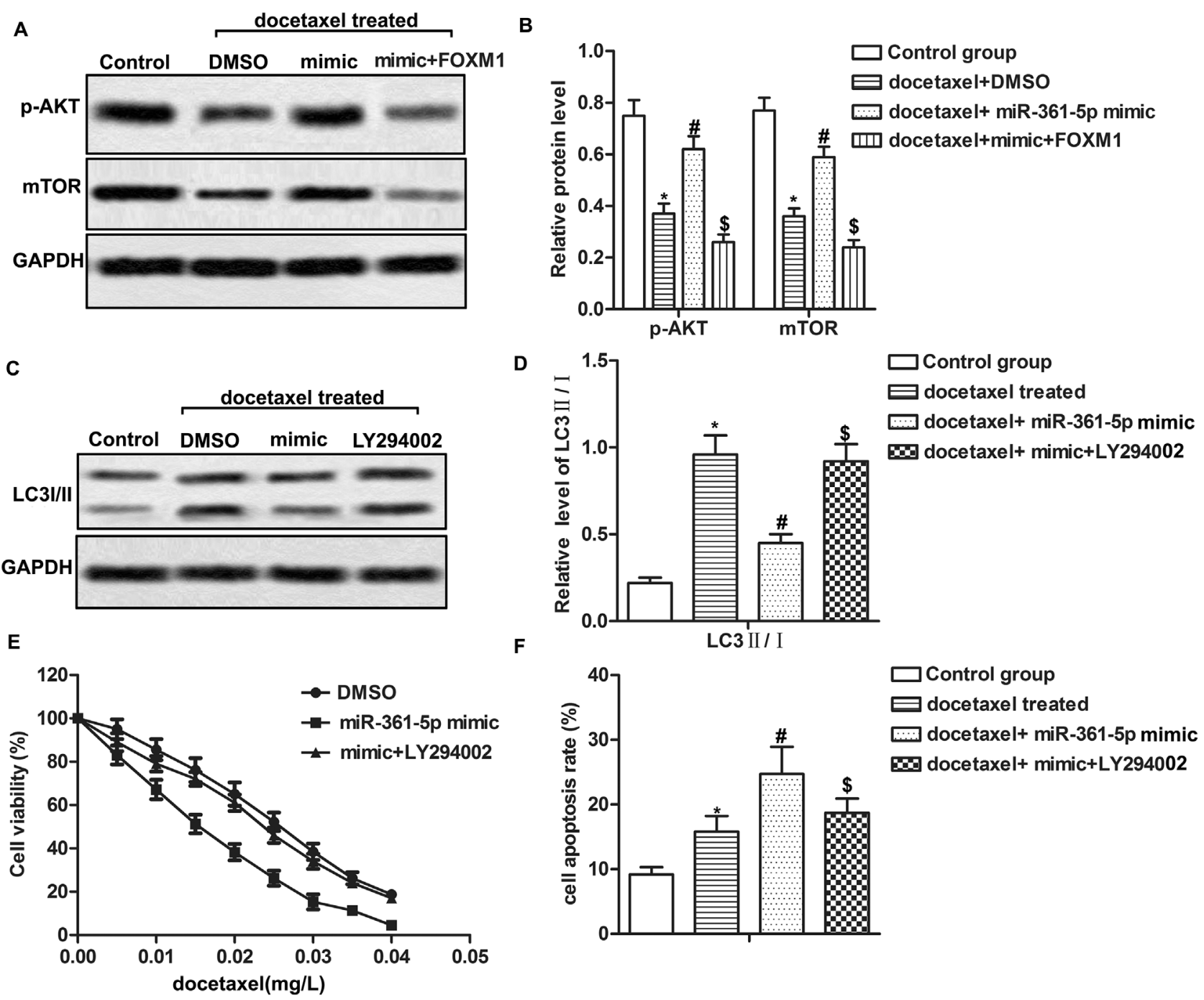

Figure 5: MiR-361-5p suppresses autophagy-induced chemoresistance via the PI3K/Akt/mTOR pathway. MiR-361-5p mimic transfected SGC-7901 cells received docetaxel treatment with or without LY294002 treatment. (A-B) Relative expression of p-AKT and mTOR was detected by western blot. GAPDH was used as an endogenous reference. (C-D) The ratio of LC3 II/I was detected by western blot. GAPDH was used as an endogenous reference. (E) Cell viability was tested by MTT assay. (F) Cell apoptosis was detected by flow cytometric analysis. The bars showed means $\pm \mathrm{SD}$ of three independent experiments. $P^{*}<0.05$ compared with control group, $P^{\#}<$ 0.05 compared with docetaxel treated group, $P^{\S}<0.05$ compared with docetaxel + miR-361-5p mimic group. 
to docetaxel of SGC-7901 and MKN-28 cells and the inhibiting effect was more obviously found in SGC-7901 cells. Our results elucidated that the inhibitive effect of miR-361-5p on chemoresistance in GC cell lines.

After learning the effect of miR-361-5p on chemoresistance of GC cells, we then set to explore its potential mechanism. Autophagy was a programmed cell survival mechanism which was reported to enhance chemoresistance of tumor cells. Hu's data showed that p53 was important for the activation of autophagy and suppression of p53 potentiated chemosensitivity in nutrient-deprived cholangiocarcinoma cells [28]. Activation of autophagy by Fusobacterium nucleatum promoted chemoresistance of colorectal cancer cells [29]. Similarly, our results showed that overexpressed miR$361-5 p$ decreased the number of $\mathrm{LC}^{+}$puncta which was widely used as a marker for autophagosomes. Moreover, the expression of Beclin-1 which was necessary for the formation of autophagosomes and the ratio of LC3 II/I were decreased while the expression of p62 which cleared the autophagosome was increased by overexpressed miR$361-5 p$, indicating that miR-361-5p inhibited autophagy in docetaxel treated SGC-7901 cells. Besides that, activation of autophagy by Rapa counteracted the inhibitory effect of miR-361-5p on chemoresistance to docetaxel through decreasing docetaxel sensibility and cell apoptosis rate in GC cells. Our data revealed that miR-361-5p suppressed chemoresistance through inhibiting autophagy in GC cells.

Furthermore, our bioinformatics study revealed that FOXM1 was one of the potential targets of miR-361-5p. FOXM1, a proliferation specific oncogenic transcription factor, was reported to regulate microtubule dynamics to mediate chemoresistance of tumor cells [20]. Previous research discovered that inhibition of FOXM1 could reverse docetaxel resistance in GC [4]. FOXM1 also mediated resistance to herceptin and paclitaxel in breast cancer [20]. Another study from Hou elucidated that overexpression of miR-361-5p suppressed lung cancer proliferation and invasion by targeting FOXM1 [30]. Consistent with previous researches, we found that the expression of FOXM1 was negatively regulated by miR-361-5p. Overexpressed FOXM1 enhanced autophagy and chemoresistance to docetaxel of GC cells, suggesting that overexpression of FOXM1 weakened the inhibitory effects of miR-361-5p on autophagy-induced chemoresistance in gastric cancer cells.

PI3K/Akt/mTOR pathway was a well-known pathway involved in the regulation of autophagy. Inhibition of $\mathrm{PI} 3 \mathrm{~K} / \mathrm{Akt} / \mathrm{mTOR}$ pathway was reported to enhance autophagy [31]. Previous study showed that autophagy was negatively regulated by the activation of mTOR in APP/PS1 double transgenic mice [32]. In accordance with these studies, overexpressed miR-361-5p was found to activate $\mathrm{PI} 3 \mathrm{~K} / \mathrm{Akt} / \mathrm{mTOR}$ pathway while overexpressed FOXM1 suppressed this pathway. Apart from that, PI3K inhibitor LY294002 abolished the effect of miR-361-5p on autophagy and chemoresistance to docetaxel of GC cells compared with docetaxel + miR-361-5p mimic group, indicating that miR-361-5p suppressed autophagy-induced chemoresistance by targeting FOXM1 via the PI3K/Akt/ mTOR pathway in GC cells.

Taken together, our present study demonstrated that miR-361-5p/FOXM1 axis played an important role in regulating chemoresistance to docetaxel of GC cells. MiR361-5p suppressed autophagy-induced chemoresistance of GC cells by targeting FOXM1 via the PI3K/Akt/mTOR pathway, providing a foundation for the mechanism research and treatment of GC.

\section{MATERIALS AND METHODS}

\section{Cell lines and culture conditions}

GC cell lines (SGC-7901, MKN-28) were purchased from American Type Culture Collection (ATCC). All the cells were cultured in DMEM (Gibco, Rockville, MD) medium supplemented with $10 \% \mathrm{FBS}$ at $37^{\circ} \mathrm{C}$.

Docetaxel (Selleckchem, Houston, TX, USA) was dissolved in DMSO and diluted to a final concentration of $0.01,0.02,0.03$ and $0.04 \mathrm{mg} / \mathrm{L}$. Rapamycin (Sigma, St. Louis, MO, USA), an autophagy activator, was used to treat cells for $24 \mathrm{~h}$ at the concentration of $100 \mathrm{nM}$. LY294002 (Selleck Inc, USA), a PI3K inhibitor, was used to treat cells for $24 \mathrm{~h}$ at the concentration of $10 \mu \mathrm{M}$.

\section{Transfection}

For miR-361-5p transfection, cells were seeded into 96 -well plate to reach $60 \%$ confluence state. MiR361-5p mimic or miR-361-5p mock (GenePharma, Shanghai, China) was transfected into SGC7901 cells using Lipofectamine 2000 reagent (Life Technologies Corporation, Carlsbad, CA, USA) according to manufacturer's protocol.

The human FOXM1 expression vector pcDNA3.1FOXM1 was transfected into cells using Lipofectamine 2000 reagent in accordance with the manufacturer's protocol.

\section{MTT assay}

For MTT assays, $5 \times 10^{3}$ cells were seeded into 96well plates in triplicate and cultured with $100 \mu \mathrm{L}$ of fresh medium which contained different concentrations of docetaxel for $48 \mathrm{~h}$. Then, MTT solution $(20 \mu \mathrm{L}, 5 \mathrm{mg} / \mathrm{ml}$ in PBS) was added to each wells and incubated at $37^{\circ} \mathrm{C}$ for $4 \mathrm{~h}$. After that, the solution was removed and $200 \mu \mathrm{L}$ of DMSO was added to each well. The optical density at $490 \mathrm{~nm}$ was measured by a microplate reader (Bio-Rad, Hercules, CA, USA) after 10 min of vibration mixing.

\section{Cell apoptosis assay}

For cell apoptosis analysis, Annexin V-FITC/ propidium iodide (PI) apoptosis detection kit 
(Multisciences, Shanghai, China) was used in our present study. Firstly, different groups of cells $\left(3 \times 10^{5}\right)$ were collected and washed in ice-cold PBS. Then cells were resuspended and incubated with $5 \mu \mathrm{L}$ of Annexin V-FIFC and $10 \mu \mathrm{L}$ of PI. Cell apoptosis was analyzed in a flow cytometer (BD Biosciences).

\section{Fluorescence microscopy}

MiR-361-5p mimic or mock transfected SGC7901 cells were transfected with GFP-LC3 plasmid by Lipofectamine $3000^{\mathrm{TM}}$ (Invitrogen). After untreated SGC7901 cells or transfected cells treated with or without docetaxel, the number of puncta formation of GFP-LC3 was determined under fluorescent microscopy as described before [33]. Cells with more than 5 puncta counted were considered to have accumulated autophagosomes.

\section{Western blot}

Proteins were extracted from cells using RIPA lysis buffer (Beyotime Institute of Biotechnology, Shanghai, China) according to the manufacturer's protocol. Total proteins were separated by SDS-PAGE gel and then transferred into PVDF membranes (Millipore). After incubating with $5 \%$ skim milk for $1 \mathrm{~h}$ at room temperature, the membranes were incubated with primary antibodies overnight at $4^{\circ} \mathrm{C}$. The primary antibodies (Cell Signaling Technology, Beverly, MA., USA) used in this study include anti-Beclin-1 (No. 4122, 1:1000), anti-LC3 I/ II (No.12741, 1:1000), anti-p62 (No.88588, 1:1000), anti-FOXM1 (No.5436, 1:1000), anti-p-AKT (No.4060, 1:2000), anti-mTOR (No.2983, 1:1000) and anti-GAPDH (No.5174, 1:2500). Then, the membranes were incubated with corresponding secondary antibodies (Cell Signaling Technology, No.7076 and NO.7074, 1:4000) at room temperature for $1 \mathrm{~h}$. ECL system (Bio-Rad Laboratories) was used for detection of antibody-bound proteins according to manufacturer's instructions.

\section{Bioinformatics method}

In order to evaluate miR-361-5p's potential target genes, the following online miRNA target prediction algorithms were used: PicTar (http://www.pictar.org/), miRanda (http://www.microrna.org/microrna/home.do), TargetScan (http://www.targetscan.org/vert_71/) and Microcosm Targets (http://www.ebi.ac.uk/enright-srv/ microcosm/htdocs/targets/v5/).

\section{Luciferase reporter assay}

The fragment of FOXM1 containing the target sequence of miR-361-5p was inserted into a pmirGlO Dual-luciferase miRNA Target Expression Vector (Promega, Madison, WI, USA) to form the reporter vector FOXM1-wild-type (FOXM1-WT) while FOXM1- mutated-type (FOXM1-MUT) contained mutated binding site. Cells at $5 \times 10^{4}$ were seeded into 96 -well plate to reached $60 \%$ confluence. Then cells were co-transfected with FOXM1-WT or FOXM1-MUT and miR-361-5p mimics using Lipofectamine 2000. The Dual-Luciferase Reporter Assay System (Promega, Madison, WI, USA) was used for testing the luciferase activity.

\section{Statistical analysis}

All results were presented as mean \pm standard deviation (SD). Student's t test was performed to analyze the difference between groups. Statistical analysis was performed with SPSS software (version 19). Values of $P<$ 0.05 were considered statistically significant.

\section{Abbreviations}

GC, gastric cancer; miRNAs, MicroRNAs; SD, standard deviation; Rapa, rapamycin.

\section{Author contributions}

All the authors conducted the conception and design of the study, acquisition and interpretation of data, drafting the article, final approval of the version to be published equally.

\section{CONFLICTS OF INTEREST}

The authors declare no conflicts of interest.

\section{FUNDING}

Supported by No. 2015020329 Natural Science Foundation of Liaoning Province of China.

\section{REFERENCES}

1. Ferlay J, Shin HR, Bray F, Forman D, Mathers C, Parkin DM. Estimates of worldwide burden of cancer in 2008: GLOBOCAN 2008. Int J Cancer. 2010; 127:2893-2917.

2. Thiel A, Ristimaki A. Gastric cancer: basic aspects. Helicobacter. 2012; 17:26-29.

3. Wagner AD, Grothe W, Haerting J, Kleber G, Grothey A, Fleig WE. Chemotherapy in advanced gastric cancer: a systematic review and meta-analysis based on aggregate data. J Clin Oncol. 2006; 24:2903-2909.

4. Li X, Yao R, Yue L, Qiu W, Qi W, Liu S, Yao Y, Liang J. FOXM1 mediates resistance to docetaxel in gastric cancer via up-regulating Stathmin. J Cell Mol Med. 2014; 18:811-823.

5. Haller DG, Misset JL. Docetaxel in advanced gastric cancer. Anticancer Drugs. 2002; 13:451-460. 
6. Yang J, Yu Y, Liu W, Li Z, Wei Z, Jiang R. Microtubuleassociated protein tau is associated with the resistance to docetaxel in prostate cancer cell lines. Res Report Urology. 2017; 9:71-77.

7. Hansen SN, Ehlers NS, Zhu S, Thomsen MB, Nielsen RL, Liu D, Wang G, Hou Y, Zhang X, Xu X, Bolund L, Yang H, Wang J, et al. The stepwise evolution of the exome during acquisition of docetaxel resistance in breast cancer cells. BMC Genomics. 2016; 17:442.

8. Peng X, Gong FM, Ren M, Ai P, Wu S, Tang J, Hu X. Proteomic analysis of docetaxel resistance in human nasopharyngeal carcinoma cells using the two-dimensional gel electrophoresis method. Anticancer Drugs. 2016; 27:748-755.

9. Wang T, Wei J, Qian X, Ding Y, Yu L, Liu B. Gambogic acid, a potent inhibitor of survivin, reverses docetaxel resistance in gastric cancer cells. Cancer Lett. 2008; 262:214-222

10. Mizushima N. The role of the Atg1/ULK1 complex in autophagy regulation. Curr Opin Cell Biol. 2010; 22:132-139.

11. Fukuda T, Oda K, Wada-Hiraike O, Sone K, Inaba K, Ikeda Y, Miyasaka A, Kashiyama T, Tanikawa M, Arimoto T, Kuramoto H, Yano T, Kawana K, et al. The anti-malarial chloroquine suppresses proliferation and overcomes cisplatin resistance of endometrial cancer cells via autophagy inhibition. Gynecol Oncol. 2015; 137:538-545.

12. Zhang P, Liu X, Li H, Chen Z, Yao X, Jin J, Ma X. TRPC5induced autophagy promotes drug resistance in breast carcinoma via CaMKKbeta/AMPKalpha/mTOR pathway. Sci Rep. 2017; 7:3158.

13. Wang KC, Chang HY. Molecular mechanisms of long noncoding RNAs. Mol Cell. 2011; 43:904-914.

14. Zeng K, Zheng W, Mo X, Liu F, Li M, Liu Z, Zhang W, Hu $\mathrm{X}$. Dysregulated microRNAs involved in the progression of cervical neoplasm. Arch Gynecol Obstetr. 2015; 292:905-913.

15. Yu H, Duan P, Zhu H, Rao D. miR-613 inhibits bladder cancer proliferation and migration through targeting SphK1. Am J Transl Res. 2017; 9:1213-1221.

16. Rajabpour A, Afgar A, Mahmoodzadeh H, Radfar JE, Rajaei F, Teimoori-Toolabi L. MiR-608 regulating the expression of ribonucleotide reductase M1 and cytidine deaminase is repressed through induced gemcitabine chemoresistance in pancreatic cancer cells. Cancer chemotherapy and pharmacology. 2017; 80:765-775.

17. Wu G, Wang J, Chen G, Zhao X. microRNA-204 modulates chemosensitivity and apoptosis of prostate cancer cells by targeting zinc-finger E-box-binding homeobox 1 (ZEB1). Am J Transl Res. 2017; 9:3599-3610.

18. Sun JJ, Chen GY, Xie ZT. MicroRNA-361-5p inhibits cancer cell growth by targeting CXCR6 in hepatocellular carcinoma. Cell Physiol Biochem. 2016; 38:777-785.
19. Wierstra I, Alves J. FOXM1, a typical proliferationassociated transcription factor. Biol Chem. 2007; 388:1257-1274.

20. Carr JR, Park HJ, Wang Z, Kiefer MM, Raychaudhuri P. FoxM1 mediates resistance to herceptin and paclitaxel. Cancer Res. 2010; 70:5054-5063.

21. Sun MY, Zhu JY, Zhang CY, Zhang M, Song YN, Rahman $\mathrm{K}$, Zhang LJ, Zhang H. Autophagy regulated by lncRNA HOTAIR contributes to the cisplatin-induced resistance in endometrial cancer cells. Biotechnol Lett. 2017; 39:1477-1484.

22. Guan H, Piao H, Qian Z, Zhou X, Sun Y, Gao C, Li S, Piao F. 2,5-Hexanedione induces autophagic death of VSC4.1 cells via a PI3K/Akt/mTOR pathway. Mol Biosyst. 2017; 13:1993-2005.

23. Tebbutt NC, Cummins MM, Sourjina T, Strickland A, Van Hazel G, Ganju V, Gibbs D, Stockler M, Gebski V, Zalcberg J. Randomised, non-comparative phase II study of weekly docetaxel with cisplatin and 5-fluorouracil or with capecitabine in oesophagogastric cancer: the AGITG ATTAX trial. Br J Cancer. 2010; 102:475-481.

24. Van Cutsem E, Moiseyenko VM, Tjulandin S, Majlis A, Constenla M, Boni C, Rodrigues A, Fodor M, Chao Y, Voznyi E, Risse ML, Ajani JA. Phase III study of docetaxel and cisplatin plus fluorouracil compared with cisplatin and fluorouracil as first-line therapy for advanced gastric cancer: a report of the V325 Study Group. J Clin Oncol. 2006; 24:4991-4997.

25. Corcoran C, Friel AM, Duffy MJ, Crown J, O'Driscoll L. Intracellular and extracellular microRNAs in breast cancer. Clin Chem. 2011; 57:18-32.

26. Zhuang ZL, Tian FM, Sun CL. Downregulation of miR361-5p associates with aggressive clinicopathological features and unfavorable prognosis in non-small cell lung cancer. Eur Rev Med Pharmacol Sci. 2016; 20:5132-5136.

27. Zhang X, Wei C, Li J, Liu J, Qu J. MicroRNA-361-5p inhibits epithelial-to-mesenchymal transition of glioma cells through targeting Twist1. Oncol Rep. 2017; 37:1849-1856.

28. Hu F, Guo XL, Zhang SS, Zhao QD, Li R, Xu Q, Wei LX. Suppression of p53 potentiates chemosensitivity in nutrient-deprived cholangiocarcinoma cells via inhibition of autophagy. Oncol Lett. 2017; 14:1959-1966.

29. Yu T, Guo F, Yu Y, Sun T, Ma D, Han J, Qian Y, Kryczek I, Sun D, Nagarsheth N, Chen Y, Chen H, Hong J, et al. Fusobacterium nucleatum promotes chemoresistance to colorectal cancer by modulating autophagy. Cell. 2017; 170:548-563.e516.

30. Hou XW, Sun X, Yu Y, Zhao HM, Yang ZJ, Wang X, Cao XC. miR-361-5p suppresses lung cancer cell lines progression by targeting FOXM1. Neoplasma. 2017; 64:526-534.

31. Saiki S, Sasazawa Y, Imamichi Y, Kawajiri S, Fujimaki T, Tanida I, Kobayashi H, Sato F, Sato S, Ishikawa K, Imoto M, Hattori N. Caffeine induces apoptosis by enhancement 
of autophagy via PI3K/Akt/mTOR/p70S6K inhibition. Autophagy. 2011; 7:176-187.

32. Wang C, Zhang X, Teng Z, Zhang T, Li Y. Downregulation of PI3K/Akt/mTOR signaling pathway in curcumin-induced autophagy in APP/PS1 double transgenic mice. Eur J Pharmacol. 2014; 740:312-320.
33. He JD, Wang Z, Li SP, Xu YJ, Yu Y, Ding YJ, Yu WL, Zhang RX, Zhang HM, Du HY. Vitexin suppresses autophagy to induce apoptosis in hepatocellular carcinoma via activation of the JNK signaling pathway. Oncotarget. 2016; 7:8452084532. https://doi.org/10.18632/oncotarget.11731. 